\title{
Neurological presentation of Fabry's disease in a 52 year old man
}

\author{
R Mohanraj, J P Leach, J C Broome, D F Smith
}

J Neurol Neurosurg Psychiatry 2002;73:340-342

Fabry's disease is an $X$ linked inborn error of metabolism due to deficient activity of the lysosomal enzyme $\alpha$ galactosidase A. Previously unrecognised Fabry's disease presenting in a 52 year old man being investigated for progressive dysarthria and ataxia is discussed. Brain magnetic resonance imaging suggested the presence of small vessel disease but skin biopsy (done to exclude cerebral autosomal dominant arteriopathy with subcortical infarcts and leucencephalopathy) showed typical changes of Fabry's disease. This diagnosis was confirmed by subsequent enzyme assays. The authors contend that Fabry's disease should be excluded, at least on clinical grounds, in patients with otherwise unexplained cerebrovascular disease.

A 52 year old man presented with a two year history of progressive slurring of speech, memory problems, and unsteadiness of gait. He also complained of involuntary twitches in both legs and occasional paraesthesiae in the fingers of one hand. On direct questioning, he admitted to urinary incontinence and erectile dysfunction. He suffered from asthma, for which he took regular inhaled salbutamol, fluticasone, salmeterol, and ipratropium bromide. Congenital lymphoedema had been diagnosed when the patient was in his 20 s and he had since been on long term diuretic treatment. He had recently been started on paroxetine for depression.

The patient was a non-smoker and was drinking a maximum of three units of alcohol a day. There was a family history of cerebrovascular disease: his mother and one of his sisters had both had a non-fatal cerebrovascular accident in their 50s. Another sister was being investigated for "visual problems". The patient had one healthy brother, but the patient's 30 year old daughter suffered from unexplained limb pains.

On examination the patient was normotensive and mildly dysarthric. No organomegaly was noted. His ocular fundi were normal and pursuits were jerky with no nystagmus. There was mild incoordination in the upper limbs. Reflexes were symmetrically normal and there was no focal deficit in power. Gait was unsteady with a broad base. The typical rash (angiokeratoma corporis diffusum) was present periumbilically, although this was not noted at initial examination. Formal neuropsychological assessment found deterioration in general intellectual and memory functions. Language and frontal lobe executive functions were well preserved.

Late onset cerebellar syndrome was diagnosed and a variety of investigations were instituted. Full blood count, urea and electrolytes, and random blood glucose results were within normal limits. The serum creatinine was initially normal but had risen to $158 \mu \mathrm{mol} / \mathrm{l}$ by the time of diagnosis. $\gamma$ Glutamyl transferase and alkaline phosphatase concentrations were modestly increased but other liver function tests were normal. Random blood alcohol was undetectable. Cerebrospinal fluid (CSF) protein concentration was increased at $0.81 \mathrm{~g} / \mathrm{l}$ but there were no cells in the CSF and immunofixation did not show oligoclonal bands.

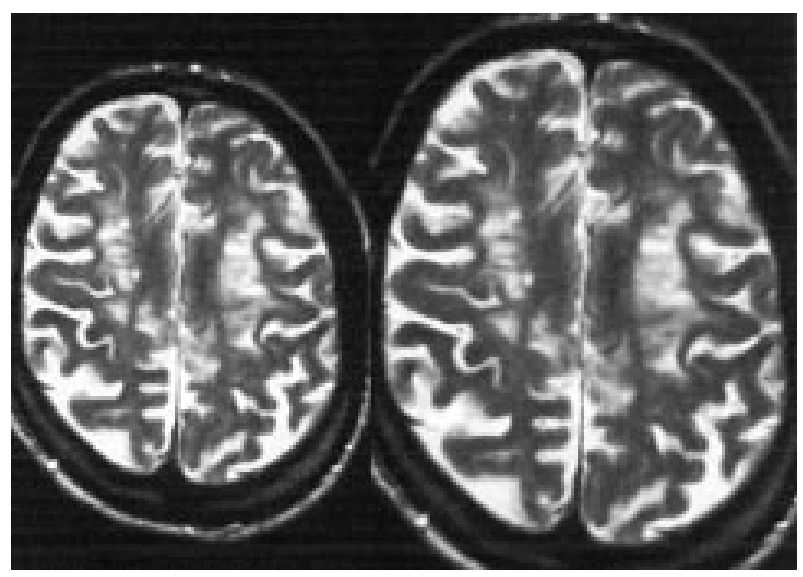

Figure 1 T2 weighted magnetic resonance images in a patient with Fabry's disease.

The following tests were normal or negative: autoantibodies (anticardiolipin antibodies, antinuclear antibody, extractable nuclear antibody, antinuclear cytoplasm antibody), lupus anticoagulant, CSF lactate, Whipple's polymerase chain reaction, mitochondrial DNA mutations (mitochondrial myopathy, encephalopathy, lactacidosis, and stroke-like episodes; myoclonus epilepsy with ragged red fibres; and a syndrome of neuropathy with ataxia and retinitis pigmentosa), serum gliadin antibody, and aminoaciduria screen.

Cranial magnetic resonance imaging showed generalised atrophy with multiple areas of high signal intensity on proton density and T2 weighted axial sections involving the white matter of both cerebellar and cerebral hemispheres (fig 1). These were thought to be areas of ischaemia or demyelination.

Nerve conduction studies showed bilateral carpal tunnel syndrome and evidence of a mild sensory motor neuropathy with reduced motor and sensory amplitudes in both lower limbs. Visual evoked responses were normal on the right and delayed on the left. An electrocardiogram showed left bundle branch block but a transthoracic echocardiogram showed no significant valve heart disease. Carotid Doppler echocardiography showed only minimal atheroma.

DNA testing for cerebral autosomal dominant arteriopathy with subcortical infarcts and leucencephalopathy (CADASIL) was negative. Skin biopsy showed abnormal small dermal blood vessels on light microscopy but no changes consistent with CADASIL. Electron microscopy of the skin showed membrane bound collections of electron dense bodies in the endothelial cells, fibroblasts, and pericytes (fig 2). The changes

Abbreviations: CADASIL, cerebral autosomal dominant arteriopathy with subcortical infarcts and leucencephalopathy; CSF, cerebrospinal fluid 

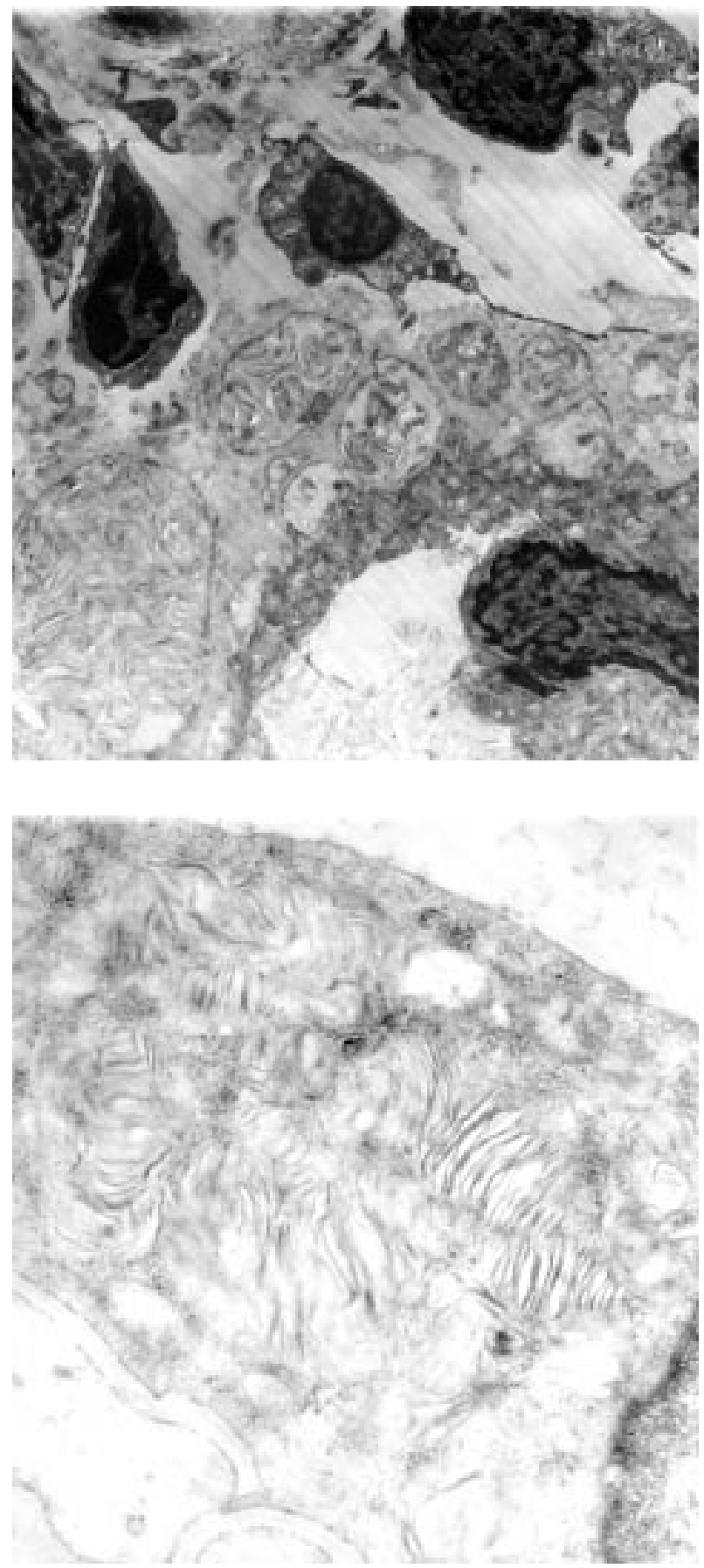

Figure 2 Electron microscopy of skin biopsy of a patient with Fabry's disease. The accumulated sphingolipids appear as intracellular striated structures. Original magnification (top) $\times 6400$, (bottom) $\times 23200$.

were thought to result from a lysosomal storage disorder. A white cell enzyme assay was carried out and this showed greatly reduced $\alpha$ galactosidase activity, confirming the diagnosis of Fabry's disease.

\section{DISCUSSION}

Fabry's disease is a lysosomal storage disorder inherited as an $\mathrm{X}$ linked recessive trait and caused by deficient activity of the lysosomal enzyme $\alpha$ galactosidase A. It has a prevalence of l/40 000 and can produce various neurological complications. Although Fabry's disease usually appears in childhood or adolescence with typical skin lesions and painful "crises", atypical affected men can present in adulthood. Most late presentations of Fabry's disease are precipitated by cardiac or renal complications; neurological presentation in adulthood remains rare. Interestingly, when our patient was questioned directly after the diagnosis, it emerged that he had been referred to a hospital clinic at the age of six with (hitherto unexplained) distal limb pains.

While the clinical signs are important, showing reduced or absent $\alpha$ galactosidase deficiency in leucocytes is essential for the diagnosis of Fabry's disease. ${ }^{1}$ The enzymatic defect leads to the systemic accumulation of neutral glycospingolipids in plasma and tissues. The lipid is stored as a myelin figure-like whorl of membranes in endothelial and smooth muscle cells, myocardium, fibroblasts, and epithelial cells of the glomerulus seen on electron microscopy. ${ }^{2}$ Most female carriers are asymptomatic but they may experience isolated acroparaesthesiae, cardiac symptoms, or a characteristic benign corneal dystrophy. Clinical manifestations in affected male patients result from a progressive disease of small vessels and may initially involve the typical rash (angiokeratoma corporis diffusum), autonomic dysfunction, and debilitating pain. Renal disease and vasculopathy of heart and brain may lead to early death in adulthood. In our patient the typical childhood presentation had been unrecognised and the rise in serum creatinine was, in retrospect, a clue to the multisystemic nature of the condition.

Progressive accumulation of ceramidetrihexoside in the intima and media of cerebral blood vessels causes ischaemic lesions in the majority of affected patients. The appearance of the lesions on magnetic resonance imaging is typical of small vessel disease. ${ }^{3}$ There is a prothrombotic tendency in patients with Fabry's disease; increased incidences of thrombophlebitis and central retinal artery occlusion have been described. ${ }^{4}$ The risk of cerebrovascular disease is increased as a result of the combination of small vessel disease and a prothrombotic tendency. Ascertaining carrier status in young women of childbearing age has obvious genetic implications.

Fabry's disease has been considered untreatable until recently but new advances in enzyme replacement therapy have raised the possibility of halting or reversing much of the multisystem damage that occurs in these patients. Treatment strategies such as gene therapy, substrate deprivation, and administration of $\alpha$ galactosidase enzyme are being evaluated. Administration of $\alpha$ galactosidase A has been shown to be safe and effective in reducing the accumulation of ceramidetrihexoside and in improving both creatinine clearance and cardiac conduction in a recently concluded randomised controlled trial. ${ }^{5}$

We suspect that the true incidence of Fabry's disease is higher than previously thought and some degree of galactosidase deficiency may underlie some cases of otherwise unexplained cerebrovascular disease. While the association of Fabry's disease with cerebrovascular disease and vascular dementia has been described in other literature, ${ }^{6}$ such a neurological presentation in midlife has not been described before. Fabry's disease should be considered in the differential diagnosis of cerebrovascular disease where other risk factors are not present.

\section{Authors' affiliations}

R Mohanraj, Research Fellow, Epilepsy Unit Western Infirmary, Glasgow G11 6NT, UK

J P Leach, J C Broome, D F Smith, Walton Centre for Neurology and Neurosurgery, Liverpool L9 7L, UK

Correspondence to: Dr J P Leach, Department of Neurology, Walton Centre for Neurology and Neurosurgery, Lower Lane, Liverpool L9 7L, UK; j.leach@liv.ac.uk

Received 1 February 2002

Accepted 27 February 2002 


\section{REFERENCES}

1 Peters FPJ, Sommer A, Vermeulen EC, et al. Fabry's disease: a

multidisciplinary disorder. Postgrad Med J 1997:73:710-2.

2 Schatzki PF, Kipreos B, Payne J. Fabry's disease: primary diagnosis by electron microscopy. Am J Surg Pathol 1979;3:211-9.

3 Crutchfield KE, Patronas NJ, Dambrosia JM, et al. Quantitative analysis of cerebral vasculopathy in patients with Fabry disease. Neurology 1998;50:1746-9.
4 Utsumi K, Yamamoto N, Kase R, et al. High incidence of thrombosis in Fabry's disease. Intern Med 1997;36:327-9.

5 Schiffmann R, Kopp JB, Austin HA 3rd, et al. Enzyme replacement therapy in Fabry's disease: a randomised control trial. JAMA 2001;285:2743-9.

6 Mendez MF, Stanley TM, Medel NM, et al. The vascular dementia of Fabry's disease. Dement Geriatr Cogn Disord 1997;8:252-7.

\section{Want full text but don't have a subscription?}

\section{Pay per view}

For just \$8 you can purchase the full text of individual articles using our secure online ordering service. You will have access to the full text of the relevant article for 48 hours during which time you may download and print the pdf file for personal use.

\section{www.jnnp.com}

